\title{
The role of serial carotid intima-media thickness assessment as a surrogate marker of atherosclerosis control in patients with recent myocardial infarction
}

\author{
Jacek Gacoń ${ }^{1,2}$, Tadeusz Przewlocki², Jakub Podolec², Rafal Badacz², Piotr Pieniazek², Wojciech Ryniewicz ${ }^{3}$, \\ Krzysztof Żmudka², Anna Kabłak-Ziembicka
}

'Department of Invasive Cardiology, E. Szczeklik's Hospital, Tarnow, Poland

${ }^{2}$ Department of Interventional Cardiology, Jagiellonian University School of Medicine, the John Paul II Hospital, Krakow, Poland

${ }^{3}$ Department of Dental Prosthetics, Institute of Dentistry, Jagiellonian University School of Medicine, Krakow, Poland

Adv Interv Cardiol 2019; 15, 1 (55): 74-80

DOI: https://doi.org/10.5114/aic.2019.81705

\begin{abstract}
A bstract
Introduction: Despite percutaneous coronary intervention ( $\mathrm{PCl}$ ), patients after their first myocardial infarction (MI) are at high risk of ischemic event recurrence. Therefore, there is a need for objective markers of adequate atherosclerosis control, independent of prescribed pharmacotherapy and patients' compliance. Such a potential indicator of major adverse cerebral and coronary event (MACCE) risk might be change in carotid intima-media thickness (CIMT), which indicates atherosclerosis growth.

Aim: To evaluate the potential associations between CIMT changes and the incidence of MACCE and recurrent MI.

Material and methods: The CIMT assessments at baseline and during 2 follow-up visits were performed in 215 patients admitted with $\mathrm{MI}$, in whom $\mathrm{PCl}$ was performed for an index lesion, followed by best medical treatment. The incidences of MACCE (cardiovascular death, recurrent MI, ischemic stroke) and new onset angina were recorded prospectively.

Results: The MACCE were recorded in 65 (30.2\%) patients and angina due to coronary lesion progression (CLP) in 27 (12.5\%) patients. Although initial CIMT values were similar in patients who suffered MACCE vs. MACCE-free patients $(1.43 \pm 0.40$ vs. 1.45 $\pm 0.44 \mathrm{~mm} ; p=0.486)$, patients in whom MACCE occurred had greater annual CIMT growth as assessed at the first ( $0.024 \pm 0.12$ vs. $0.009 \pm 0.16 \mathrm{~mm} /$ year; $p<0.001)$ and subsequent follow-up visit $(0.050 \pm 0.1 \mathrm{vs}$. $0.001 \pm 0.1 \mathrm{~mm} /$ year; $p<0.001)$, in mean $36.5 \pm 29.3$ and $53.3 \pm 37.1$ months, respectively. An optimal cut-off value for annual CIMT change of $>0.003 \mathrm{~mm} /$ year (sensitivity: $84.5 \%$, specificity: 49.3\%) for MI plus CLP (AUC = 0.673) occurred an independent indicator of MACCE (HR = 3.00; 95\% Cl: 1.496-6.016), recurrent MI ( $\mathrm{HR}=4.59,95 \% \mathrm{Cl}: 1.591-13.217)$, and MI plus CLP (HR = 3.50, 95\% Cl: 1.759-6.964).
\end{abstract}

Conclusions: Annual CIMT change might be a potentially valuable marker of atherosclerosis response to post-MI treatment.

Key words: carotid intima-media thickness, coronary artery diseases, symptom recurrence, myocardial infarction, atherosclerosis progression, major adverse cerebral and coronary events.

Su m m a ry

There is a need for direct indicators of atherosclerotic process slowing or growth. Baseline carotid intima-media thickness (CIMT) is independently associated with extent of atherosclerosis and ischemic cardiovascular events. Little is known about whether CIMT change may address atherosclerosis growth in individual patients, and as such whether CIMT change may be related to risk of major adverse cerebral and coronary event (MACCE) risk. Therefore, the present study aimed to evaluate the potential associations between CIMT change and the incidence of MACCE. Repeated assessment of CIMT changes might serve as a reflector of achieved atherosclerosis control during follow-up as it predicts the risk of major adverse cerebral and coronary event, recurrent myocardial infarction, and coronary disease progression.

\section{Corresponding author:}

Anna Kabłak-Ziembicka MD, PhD, Department of Interventional Cardiology, Jagiellonian University School of Medicine, John Paul II Hospital, Krakow, Poland, phone: +48 12614 3501, fax: +48 12614 2790, e-mail: kablakziembicka@op.pl

Received: 16.10.2018, accepted: 9.12.2018. 


\section{Introduction}

Despite percutaneous coronary intervention $(\mathrm{PCI})$, cardiac rehabilitation and best medical therapy (BMT), survivors of first acute myocardial infarction (MI) are at high risk of a recurrent $\mathrm{MI}$, or a subsequent vascular event and cardiovascular death (CVD) [1-3].

Most studies of post-MI outcomes focus on the acute phase after the index event, with few data available for follow-up beyond the first year. Although the risk of cardiovascular events is highest in the first year after the index MI, it remains remarkable in subsequent years [4]. The relative risk for all-cause death and cardiovascular outcomes (recurrent MI, CVD) is at least 30\% higher than that in a general reference population at both $1-3$ years and 3-5 years after MI [5]. It is true particularly for patients with additional risk factors such as diabetes, hypertension, or older age.

Furthermore, patients despite prescribed medications often demonstrate poor compliance, or the dosages prescribed are generally $50 \%$ or less of the dosages used in clinical trials [6, 7]. Both medication adherence and therapeutic goals are achieved in a minority of patients [6-8].

The data reinforce the importance of secondary prevention in improving long-term prognosis of post-MI patients. However, only a few, but indirect, prognostic tools are available, for instance assessment of the left ventricle function, biochemical markers of heart failure (e.g. NT-pro-BNP) or inflammatory process activity (e.g. hs-CRP), and occlusive stenotic lesions in extra-coronary vascular beds [9-12].

Yet, there is a need for direct indicators of atherosclerotic process slowing or growth, independent of prescribed pharmacotherapy and patients' compliance. A potential indicator of cardiovascular risk might be carotid intima-media thickness (CIMT). Although baseline CIMT is independently associated with the extent of atherosclerosis and ischemic cardiovascular events $[13,14]$, little is known about whether CIMT change may address atherosclerosis growth in other than carotid artery territory in individual patients, and as such whether CIMT change may be related to risk of major adverse cerebral and coronary events (MACCE), recurrent MI or recurrent angina.

\section{Aim}

Therefore, the present study aimed to evaluate the potential associations between CIMT change and the incidence of MACCE, recurrent $\mathrm{MI}$ and new angina due to coronary lesion progression (CLP) in patients who underwent MI.

\section{Material and methods}

This prospective study comprised 215 patients (154 male; mean age: $63.3 \pm 9.8$ years) admitted with recent $\mathrm{MI}$, in whom $\mathrm{PCl}$ was performed for an index lesion. Of note, patients were treated according to contemporary guidelines concerning coronary intervention as well as best medical treatment (BMT) afterwards.

The CIMT assessments at baseline and during at least 2 follow-up visits were performed in all patients. The incidences of MACCE, CVD, recurrent MI, ischemic stroke (IS) and new onset angina were recorded prospectively for a median of 60 months.

Inclusion criteria were as follows: age over 18 years, acute $\mathrm{MI}$ with confirmed significant (at least $50 \%$ lumen reduction) stenosis in an index coronary artery.

Exclusion criteria: chronic or acute inflammatory status, active cancer, critical state, acute heart failure or with congestive heart failure in NYHA classes IV, patients not willing to participate in the present study, or suboptimal carotid artery ultrasound (e.g. massive calcifications enabling CIMT assessment).

According to current guidelines, patients were prescribed dual antiplatelet therapy and other medications, including typically a statin, $\beta$-blocker, and angiotensin converting enzyme inhibitors (ACEI)/angiotensin receptor blockers (ARB), as per guidelines of the European Society of Cardiology [15].

All subjects gave their informed consent prior to enrolment in accordance with the requirements of the institutional local Ethics Committee. The study was performed consistently with the requirements of the Declaration of Helsinki.

\section{Carotid intima-media measurement}

Carotid ultrasonography was done with high-resolution B-mode, color Doppler, and pulsed wave Doppler ultrasonography, using a Toshiba Aplio 300 ultrasound machine (Toshiba Medical Systems Co) equipped with a 4-12-MHz linear array transducer.

During hospital stay (baseline examination), all patients underwent measurement of the maximum carotid intima-media complex of the near and far walls at 3 segments, including the distal $1 \mathrm{~cm}$ of the common carotid artery, at the bifurcation, and at the level of the internal carotid artery. All measurements were expressed as mean maximum CIMT.

After initial CIMT assessment, participants were followed up twice with CIMT re-evaluation at a mean of 3 and 4.5 years.

The annual change in CIMT (mm/year) was calculated using the following equation: annual change of CIMT = (follow-up CIMT - baseline CIMT)/observation period in years.

The difference between the follow-up and baseline CIMT was also expressed as an absolute $\Delta$ value according to the following equation: $\triangle \mathrm{CIMT}=$ follow-up CIMT - baseline CIMT.

\section{Follow-up period and cardiovascular events}

The clinical follow-up was continued beyond the last CIMT re-assessment in order to establish the potential 
relationship between CIMT change and MACCE, recurrent $\mathrm{MI}$ and new onset of angina for a median of 60 months.

Recurrent MI was diagnosed according to contemporary criteria of the European Society of Cardiology [16]. The diagnosis of IS was established by a neurologist to ensure validity. The CVD was defined as fatal IS, fatal MI, or other CVD (i.e., any sudden or unexpected death unless proven as non-cardiovascular on autopsy). The relationship between new onset angina and coronary status was established with coronary angiography in order to ensure coronary lesion progression, and exclude symptoms related to stenosis recurrence (restenosis or stent thrombosis as related to different mechanisms) [17, 18]. For further analysis only new angina due to CLP was taken into account.

\section{Statistical analysis}

Student's t test was used for comparison of continuous variables, and the $\chi^{2}$ test was used to compare proportions of categorical variables. Means of the analyzed parameters across groups were tested by analysis of variance test. Frequencies were compared by the $\chi^{2}$ test for independence.

The influence of CIMT change, clinical, and biochemical factors on the incidence of the endpoints MACCE, recurrent MI, and new angina due to CLP was assessed with Cox univariate hazard analysis, and in cases with a $p$-value $<0.05$, they were included in a multivariate stepwise Cox proportional hazards analysis. The results of the multivariate Cox proportional hazards analysis were expressed as hazard ratio (HR) and 95\% confidence interval $(\mathrm{Cl})$. Kaplan-Meier analysis for recurrent $\mathrm{MI}$ and new angina due to coronary lesion progression according to quartiles (A lowest; D - highest quartile) of the annual CIMT change on the final ultrasonography was performed.

Receiver operating characteristic (ROC) curves were constructed to establish the best cut-off value for the annual CIMT progression rate predicting risk of MACCE, recurrent MI and CLP. The area under the ROC curve (AUC) was calculated. Statistical analyses were performed with Statistica version 12.0 software (StatSoft, Inc, Tulsa, OK). $P<0.05$ was considered statistically significant.

\section{Results}

During the median 60-month follow-up period, MACCE were recorded in 65 (30.2\%) patients, including 10 CVDs (9 Mls/sudden cardiac, 1 IS), 42 non-fatal MIs and/or 15 non-fatal ISs. Recurrent MI occurred in 12 (5.6\%) subjects during first year of follow-up, and in 26 (12.1\%) at 3 years. Additionally, angina recurrence in CCS classes II-III was noted in 30 (14\%) patients, of which new angina due to CLP was observed in 20 (9.3\%) patients, while angina due to in-stent restenosis was observed in 10 (4.7\%), as evidenced with coronary angiography.
The detailed clinical characteristics and assessment depending on MACCE plus CLP incidence are summarized in Table I. Subjects in whom MACCE plus CLP occurred, as compared to the MACCE-free subgroup, were more often hypertensive $(p=0.026)$, had higher levels of serum creatinine $(p=0.049)$ and hs-CRP $(p=0.001)$, and had a higher mean number of cardiovascular risk factors among hypertension, hyperlipidemia, diabetes, and cigarette smoking $(p=0.058)$ (Table $\mathrm{I}$ ).

Initial CIMT values were similar in patients who suffered MACCE and event-free patients $(1.43 \pm 0.40 \mathrm{~mm}$ vs. $1.45 \pm 0.44 \mathrm{~mm} ; p=0.486$ ).

At first CIMT reassessment during the mean follow-up period of $36.5 \pm 29.3$ months, patients in whom MACCE occurred had 2.7-fold greater annual CIMT growth as compared to the event-free subset of patients $(0.024 \pm 0.12$ $\mathrm{mm}$ vs. $0.009 \pm 0.16 \mathrm{~mm} /$ year; $p<0.001)$. Similar findings concerned the final CIMT reassessment performed during the mean follow-up period of $53.3 \pm 37.1$ months ( 0.050 $\pm 0.1 \mathrm{~mm}$ vs. $0.001 \pm 0.1 \mathrm{~mm} /$ year; $p<0.001$, respectively).

In patients in whom annual CIMT change showed clear carotid atherosclerosis reduction ( $1^{\text {st }}$ quartile for annual CIMT change), a significantly lower number of recurrent $\mathrm{MI}$ and new angina due to CLP was observed, as compared to subjects with annual CIMT changes in the $2^{\text {nd }}, 3^{\text {rd }}$ and $4^{\text {th }}$ quartiles ( $p=0.008$ by log rank test), and as compared to the $4^{\text {th }}$ quartile $(p<0.001$ by log rank test) (Figure 1). The Kaplan-Meier free-survival curves were $100,97.8$ and $88.4 \%$ at 1,3 and 5 years for the $1^{\text {st }}$ quartile, while they were $92.6,76.6$ and $61.0 \%$ for the $4^{\text {th }}$ quartile, respectively (Figure 1).

Cox univariate regression analysis indicated several factors possibly associated with cardiovascular event risk, of which a multivariate stepwise Cox proportional hazards analysis identified that risk of MACCE was associated independently with the annual CIMT progression $>0.003 \mathrm{~mm} /$ year and serum creatinine level (Table II). Recurrent MI, as well as recurrent $\mathrm{MI}$ and new angina due to CLP, was associated with the annual CIMT progression $>0.003 \mathrm{~mm} /$ year, serum creatinine level, and history of hyperlipidaemia (Table II).

The CIMT change was found to be an independent indicator of MACCE, recurrent $\mathrm{MI}$ and $\mathrm{MI}$ plus CLP with an optimal cut-off value of CIMT progression rate of $>0.003 \mathrm{~mm}$ /year for all study end-points (Figures $2 \mathrm{~A}-\mathrm{C}$ ). Although the annual CIMT progression rate of $0.003 \mathrm{~mm} /$ year was the best predictor of MACCE, recurrent $\mathrm{MI}$ and CLP, associated with fairly good sensitivity (between $80 \%$ and $84.5 \%$ ), it demonstrated only mild specificity, between $47 \%$ and $49.3 \%$ (AUC between 0.638 and 0.673 ) (Table III).

\section{Discussion}

In the first decade of the twenty-first century, in survivors of first $\mathrm{MI}$, the cumulative incidence of death was 
Table I. Baseline characteristics of the study group

\begin{tabular}{|c|c|c|c|c|}
\hline Parameter & $\begin{array}{l}\text { Study group } \\
\quad(N=215)\end{array}$ & $\begin{array}{l}\text { Subjects with } \\
\text { MACCE plus CLP } \\
(n=83)\end{array}$ & $\begin{array}{l}\text { MACCE-free } \\
\text { subjects } \\
(n=132) \\
\end{array}$ & $P$-value \\
\hline Age, mean (SD) [years] & $63.3(9.8)$ & $63.1(9.2)$ & $61.3(10.1)$ & 0.396 \\
\hline Men, $n(\%)$ & $154(71.6)$ & $59(71)$ & $95(72)$ & 0.888 \\
\hline Hypertension, $n(\%)$ & $199(93)$ & $81(98)$ & $118(89)$ & 0.026 \\
\hline Diabetes mellitus, $n(\%)$ & $102(47)$ & $42(51)$ & $60(45)$ & 0.462 \\
\hline Hyperlipidemia, $n$ (\%) & $194(90)$ & $76(92)$ & $118(89)$ & 0.601 \\
\hline Smoking history, $n(\%)$ & $138(64)$ & $57(69)$ & $81(61)$ & 0.276 \\
\hline $\begin{array}{l}\text { Number of cardiovascular risk factors among hypertension, } \\
\text { hyperlipidemia, diabetes, cigarette smoking, mean (SD) }\end{array}$ & $2.93(0.88)$ & $3.05(0.78)$ & $2.86(0.93)$ & 0.058 \\
\hline Statin, $n(\%)$ & $210(98)$ & $80(96)$ & $130(98.5)$ & 0.320 \\
\hline Intermittent claudication, $n$ (\%) & $66(31)$ & $31(37)$ & $35(26)$ & 0.093 \\
\hline LVEF, mean (SD) \% & $52.8(10.1)$ & $52.7(10.5)$ & $52.3(9.9)$ & 0.442 \\
\hline hsCRP, mean (SD) [g/l] & $3.12(2.79)$ & $3.25(3.38)$ & $3.06(2.31)$ & 0.001 \\
\hline Body mass index, mean (SD) $\left[\mathrm{kg} / \mathrm{m}^{2}\right]$ & $27.6(3.6)$ & $27.2(3.8)$ & $27.8(3.4)$ & 0.494 \\
\hline Creatinine, mean (SD) $[\mu \mathrm{mol} / \mathrm{l}]$ & $93(33.4)$ & $96.9(36.7)$ & $90.4(27.3)$ & 0.049 \\
\hline Total cholesterol, mean (SD) [mmol/ll] & $4.97(1.31)$ & $5.07(1.41)$ & $4.9(1.23)$ & 0.486 \\
\hline LDL cholesterol, mean (SD) [mmol/ll] & $3.06(1.1)$ & $3.08(1.1)$ & $3.05(1.1)$ & 0.255 \\
\hline HDL cholesterol, mean (SD) [mmol/l] & $1.19(0.33)$ & $1.21(0.36)$ & $1.17(0.31)$ & 0.277 \\
\hline Triglycerides, mean (SD) [mmol/l] & $1.74(1.43)$ & $1.97(1.8)$ & $1.6(1.1)$ & 0.217 \\
\hline
\end{tabular}

$C A D$ - coronary artery disease, IS - ischemic stroke, LVEF - left ventricular ejection fraction, $M I-$ myocardial infarction.

$12.1 \%$ at 1 year and $17.5 \%$ at 3 years, while recurrent MI were $5 \%$ and $8.2 \%$, respectively [3]. In the years between 2004 and 2010, in England, in all survivors of a first acute $\mathrm{MI}$, the risk of a second $\mathrm{MI}$ was highest during the first year and the cumulative risk increased more gradually thereafter [1]. For men, 1- and 7-year cumulative risks were $5.6 \%$ and $13.9 \%$; for women, they were $7.2 \%$ and $16.2 \%$ [1]. Data from Swedish national registries of 108315 patients admitted to hospital with a primary MI between the years 2006 and 2011 demonstrated that the composite endpoint of non-fatal MI, non-fatal IS or CVD was $18.3 \%$ during the first year after the index $\mathrm{MI}$ [2].

In the present study, the 5-year MACCE incidence was $30.2 \%$, and including CLP 38.6\%. Recurrent MI occurred in $5.6 \%$ of subjects during the first year of follow-up, and in $12.1 \%$ at 3 years. Thus, despite the attempt to implement BMT according to guidelines, our data are consistent with others in terms of MACCE incidence.

Moreover, in patients who suffered from MACCE, we found a 2.7-fold greater annual CIMT progression rate as compared to the event-free subgroup of patients. Our study indicated that the annual CIMT progression rate of $0.003 \mathrm{~mm} /$ year is a sensitive marker of risk for MACCE, recurrent $\mathrm{MI}$ and CLP (sensitivity between $80 \%$ and $84.5 \%$ ), but it demonstrated only mild specificity between $47 \%$ and $49.3 \%$ (AUC between 0.638 and 0.673 ).

In line with our findings, in the CLAS trial, patients with an annual common CIMT progression rate of 0.034

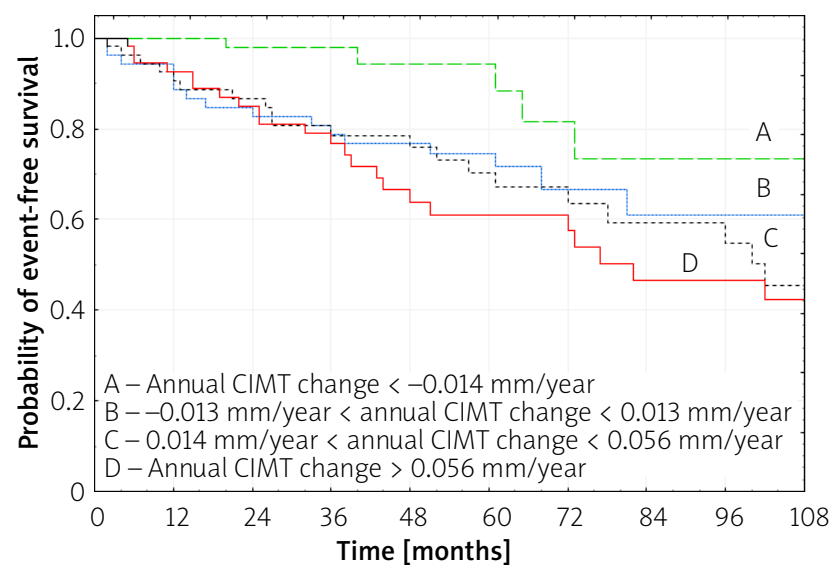

Figure 1. Kaplan-Meier analysis for recurrent $\mathrm{MI}$ and new angina due to coronary lesion progression according to quartiles ( $\mathrm{A}$ - lowest; $\mathrm{D}$ - highest quartile) of the annual CIMT change on the final ultrasonography ( $p<0.001$ for A vs. D by log-rank test; $p=0.008$ for A vs. B, C, D by log-rank test) 
Table II. Multivariate stepwise Cox proportional hazards analysis. Independent risk factors of MACCE, recurrent $\mathrm{MI}$ and recurrent $\mathrm{MI}$ plus coronary lesion progression (CLP)

\begin{tabular}{lccc} 
Cardiovascular event & Hazard ratio & 95\% confidence interval & $P$-value \\
\hline MACCE: & & & \\
\hline Serum creatinine level & 1.01 & $1.003-1.017$ & 0.006 \\
\hline The annual CIMT progression $>0.003 \mathrm{~mm} /$ year & 3.00 & $1.496-6.016$ & $<0.001$ \\
\hline Recurrent MI: & & & \\
\hline Serum creatinine level & 1.01 & $1.003-1.017$ & 0.004 \\
\hline Hyperlipidemia & 3.15 & $1.271-7.784$ & 0.013 \\
\hline Annual CIMT progression $>0.003 \mathrm{~mm} /$ year & 4.59 & $1.591-13.217$ & 0.005 \\
\hline Recurrent MI plus new angina due to CLP: & & & \\
\hline Serum creatinine level & 1.01 & $1.004-1.018$ & 0.002 \\
\hline Hyperlipidemia & 3.50 & $1.759-6.964$ & 0.005 \\
\hline Annual CIMT progression $>0.003 \mathrm{~mm} /$ year & 2.95 & $1.378-6.323$ & $<0.001$
\end{tabular}

CIMT-carotid intima-media thickness, CLP-coronary lesion progression, MACCE - major adverse cerebral and coronary events, MI - myocardial infarction.

$\mathrm{mm} /$ year had a 2.9-fold higher CVD risk compared to those with a common CIMT progression rate of 0.011 $\mathrm{mm}$ /year or less [19].

Consistent with our results, Okayama et al. observed that a higher median progression rate of common CIMT (0.03 mm/year) was a significant determinant of MACCE $(\mathrm{HR}=2.24 ; 95 \% \mathrm{Cl}: 1.25-4.03, p<0.01)$ during a mean follow-up of 7.6 years [20]. Controversially, in the IMPROVE study, assessment of 15-month progression demonstrated that only the fastest CIMT max-progression was significantly associated with risk of subsequent vascular events [21].

We demonstrated that when patients were stratified into quartiles based on the annual change in CIMT, patients in the $1^{\text {st }}$ quartile had a lower probability of recurrent $\mathrm{MI}$ and CLP, as compared to patients in the other quartiles. This finding is in line with a study of Hirano et al., including 240 patients with stable coronary artery disease [22]. Furthermore, Hirano et al. found that addition of the change in plaque-CIMT max in the follow-up period of 6 months, to conventional risk factors, resulted in a greater area under the ROC curve compared with conventional Framingham risk factors alone (0.81 and 0.70 , respectively, $p=0.02$ ) [22].

The idea of atherosclerosis tracking through serial CIMT assessments in patients with cardiovascular risk factors in the primary and the secondary prevention populations is very attractive as objective indicators of achieved atherosclerosis control would improve health outcome [23].

Many data report poor compliance and frequent underdosage of medications in patients discharged for MI. Korhonen et al. found that only $49 \%$ of the patients adhered ( $\geq 80 \%$ of days covered) to all 3 medications including ACE inhibitors/ARBs, $\beta$-blockers, and statins [24]. It is estimated that after 1,3 , and 5 years, 78, 64, and $58 \%$ of survivors who had started therapy were still receiving $\beta$-blockers, 86,78 , and $74 \%$ were receiving ACE-inhibitors, and 85,80 , and $82 \%$ were receiving statins, respectively [7]. Furthermore, the dosages prescribed were generally $50 \%$ or less of the dosages used in clinical trials, and dosages did not increase during the observation period [7]. Patients who did not start treatment shortly after discharge had a low probability of starting treatment later [7].

Moreover, 6 months after an MI, even in patients with a high adherence to drug treatments ( $\geq 90 \%$ of days covered), blood pressure $<140 / 90 \mathrm{~mm} \mathrm{Hg}$, low-density lipoprotein (LDL) $<100 \mathrm{mg} / \mathrm{dl}$ (in patients on statins), $\mathrm{HbA}_{1 \mathrm{c}}$ $<7 \%$ (in treated diabetics), and smoking persistence were observed in $74 \%, 76 \%, 45 \%$, and $27 \%$ of patients, respectively [25].

As we found in the present study, CIMT change over time might serve as an objective marker of atherosclerosis control, and as such, without much knowledge on patients' drug adherence and achieved control of cardiovascular risk factors, might serve as an additional tool to encourage lifestyle modification and motivational education programs to reduce risk of cardiovascular events.

\section{Study limitations}

The period of CIMT measurement intervals was not pre-defined. Only baseline risk factor levels and medications were used for all analyses.

\section{Conclusions}

Repeated assessment of CIMT changes might serve as a reflector of achieved atherosclerosis control during follow-up as it predicts the risk of MACCE, recurrent MI, and coronary disease progression.

\section{Conflict of interest}

The authors declare no conflict of interest. 
A

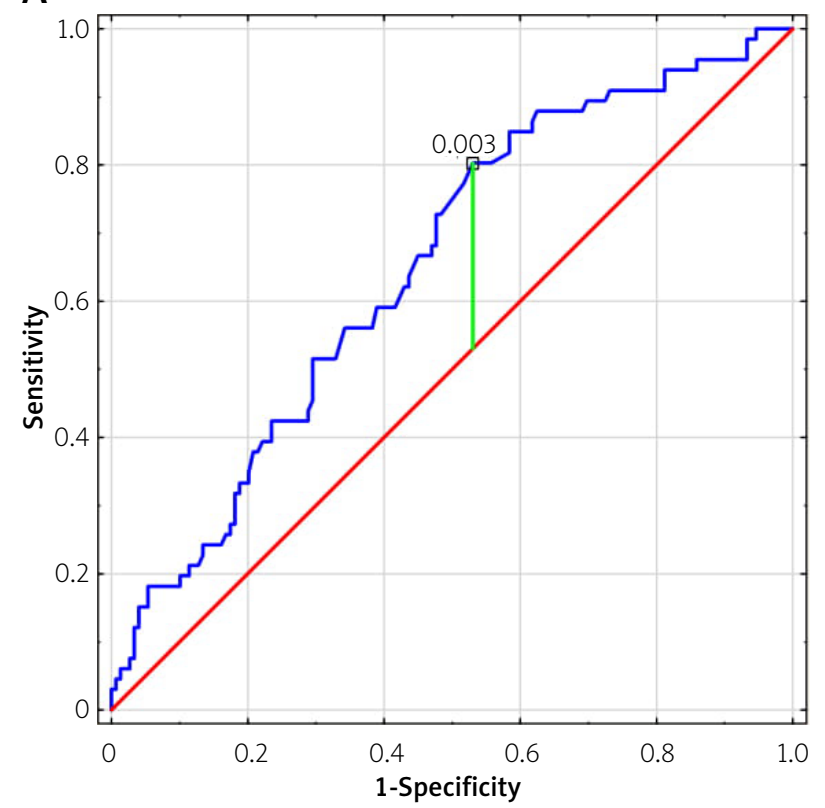

C

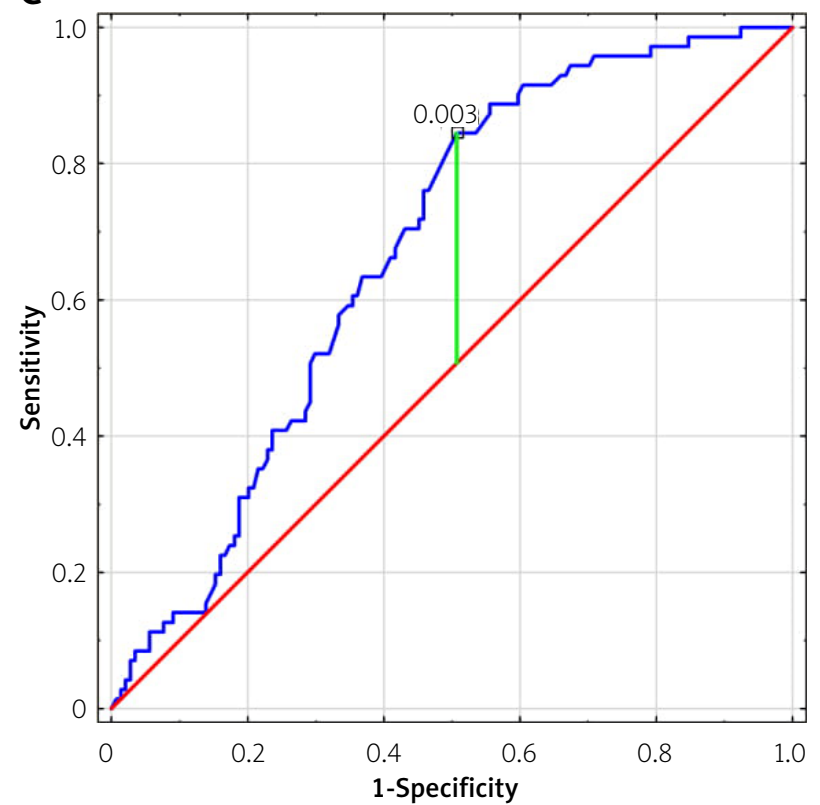

B

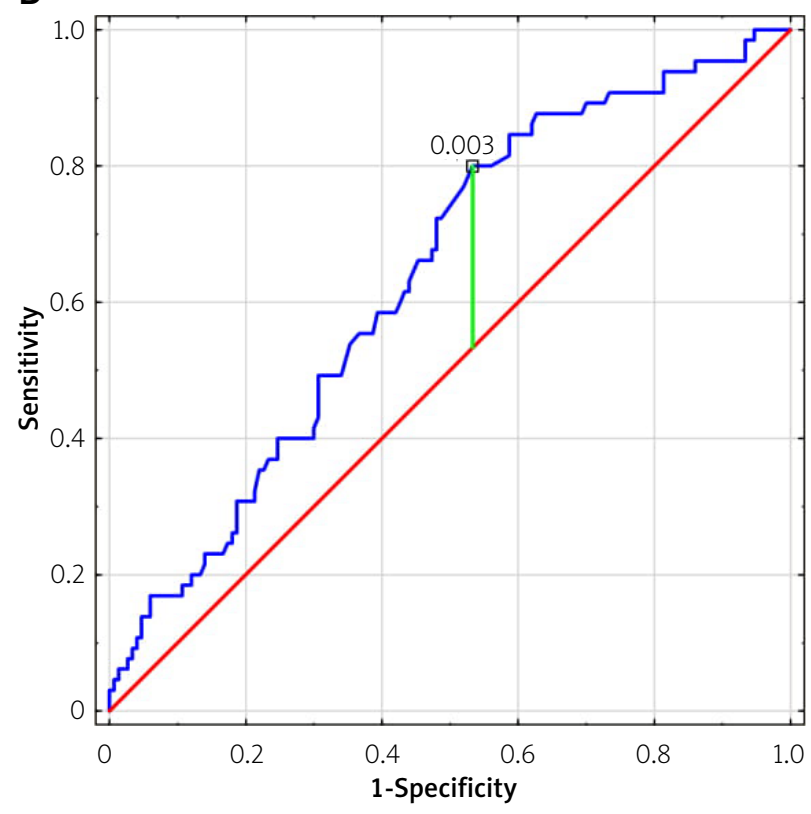

Figure 2. Receiver operating characteristics (ROC) curves for annual CIMT progression rate cut-offs for MACCE (A), recurrent MI (B) and CAD progression $(C$; recurrent $\mathrm{MI}$ plus new angina for coronary lesion progression)

Table III. Receiver operating characteristic (ROC) curves for the optimal annual CIMT progression rate cut-off value to predict risk of MACCE, recurrent MI and CLP

\begin{tabular}{lccccc} 
Event & $\begin{array}{c}\text { Annual CIMT progression } \\
{[\mathrm{mm} / \text { year] }}\end{array}$ & AUC & 95\% Cl & Sensitivity (\%) & Specificity (\%) \\
\hline MACCE & 0.003 & 0.651 & $0.574-0.728$ & 80.3 & 47 \\
\hline Recurrent MI & 0.003 & 0.683 & $0.561-0.716$ & 80 & 47.6 \\
\hline Recurrent MI plus CLP & 0.003 & 0.673 & $0.601-0.744$ & 84.5 & 49.3
\end{tabular}

CIMT-carotid intima-media thickness, CLP-coronary lesion progression, MACCE-major adverse cerebral and coronary events, MI-myocardial infarction. 


\section{References}

1. Smolina K, Wright FL, Rayner M, et al. Long-term survival and recurrence after acute myocardial infarction in England, 2004 to 2010. Circ Cardiovasc Qual Outcomes 2012; 5: 532-40.

2. Jernberg T, Hasvold P, Henriksson M, et al. Cardiovascular risk in post-myocardial infarction patients: nationwide real world data demonstrate the importance of a long-term perspective. Eur Heart J 2015; 36: 1163-70.

3. Campo G, Saia F, Guastaroba P, et al. Prognostic impact of hospital readmissions after primary percutaneous coronary intervention. Arch Intern Med 2011; 171: 1948-9.

4. Rapsomaniki E, Thuresson M, Yang E, et al. Using big data from health records from four countries to evaluate chronic disease outcomes: a study in 114364 survivors of myocardial infarction. Eur Heart I Qual Care Clin Outcomes 2016; 2: 172-83.

5. Johansson S, Rosengren A, Young K, et al. Mortality and morbidity trends after the first year in survivors of acute myocardial infarction: a systematic review. BMC Cardiovasc Disord 2017; 17: 53.

6. Lee YM, Kim RB, Lee HJ, et al. Relationships among medication adherence, lifestyle modification, and health-related quality of life in patients with acute myocardial infarction: a cross-sectional study. Health Qual Life Outcomes 2018; 16: 100.

7. Gislason GH, Rasmussen JN, Abildstrøm SZ, et al. Long-term compliance with beta-blockers, angiotensin-converting enzyme inhibitors, and statins after acute myocardial infarction. Eur Heart J 2006; 27: 1153-8.

8. Urbinati S, Olivari Z, Gonzini L, et al. Secondary prevention after acute myocardial infarction: drug adherence, treatment goals, and predictors of health lifestyle habits. The BLITZ-4 Registry. Eur J Prev Cardiol 2015; 22: 1548-56.

9. Gacoń J, Badacz R, Stepien E, et al. Diagnostic and prognostic micro-RNAs in ischaemic stroke due to carotid artery stenosis and in acute coronary syndrome: a four-year prospective study. Kardiol Pol 2018; 76: 362-9.

10. Przewlocki T, Kablak-Ziembicka A, Tracz W, et al. Renal artery stenosis in patients with coronary artery disease. Kardiol Pol 2008; 66: 856-64.

11. Cannon CP, Greenberg BH. Risk stratification and prognostic factors in the post-myocardial infarction patient. Am J Cardiol 2008; 102: 13G-20G.

12. Badacz R, Przewlocki T, Gacon J, et al. Circulating miRNA levels differ with respect to carotid plaque characteristics and symptom occurrence in patients with carotid artery stenosis and provide information on future cardiovascular events. Adv Interv Cardiol 2018; 14: 75-84.

13. Wrotniak L, Kablak-Ziembicka A, Karch I, et al. Multiterritory atherosclerosis and carotid intima-media thickness as cardiovascular risk predictors after percutaneous angioplasty of symptomatic subclavian artery stenosis. J Ultrasound Med 2016; 35: 1977-84.

14. Bryniarski KL, Tokarek T, Bryk T, et al. Intima-media thickness and ankle-brachial index are correlated with the extent of coronary artery disease measured by the SYNTAX score. Adv Interv Cardiol 2018; 14: 52-8.

15. Montalescot G, Sechtem U, Achenbach S, et al. ESC guidelines on the management of stable coronary artery disease. The Task Force on the management of stable coronary artery disease of the European Society of Cardiology. Eur Heart J 2013; 34: 29493003.
16. Thygesen K, Alpert JS, Jaffe AS, et al. Third universal definition of myocardial infarction. Eur Heart J 2012; 33: 2551-67.

17. Tekieli L, Pieniazek P, Musialek P, et al. Zotarolimus-eluting stent for the treatment of recurrent, severe carotid artery in-stent stenosis in the TARGET-CAS population. J Endovasc Ther 2012; 19: 316-24.

18. Briede I, Narbute I, Kumsars I, et al. Two-year clinical outcomes after implantation of an everolimus-eluting bioresorbable scaffold (Absorb) in stable angina and acute coronary syndrome patients. Single-centre real-life registry data. Adv Interv Cardiol 2018; 14: 144-8.

19. Hemphill LC. The Cholesterol Lowering Atherosclerosis Study (CLAS): what it tells us about niacin/colestipol therapy. J Clin Lipidol 2015; 9: 11-3.

20. Okayama KI, Mita T, Gosho M, et al. Carotid intima-media thickness progression predicts cardiovascular events in Japanese patients with type 2 diabetes. Diabetes Res Clin Pract 2013; 101: 286-92.

21. Baldassarre D, Hamsten A, Veglia F, et al. Measurements of carotid intima-media thickness and of interadventitia common carotid diameter improve prediction of cardiovascular events: results of the IMPROVE (carotid intima media thickness [IMT] and IMT-progression as predictors of vascular events in a high risk European population) study. I Am Coll Cardiol 2012; 60: 1489-99.

22. Hirano M, Nakamura T, Kitta Y, et al. Short-term progression of maximum intima-media thickness of carotid plaque is associated with future coronary events in patients with coronary artery disease. Atherosclerosis 2011; 215: 507-12.

23. Gacoń J, Przewłocki T, Podolec J, et al. Prospective study on the prognostic value of repeated carotid intima-media thickness assessment in patients with coronary and extra coronary steno-occlusive arterial disease. Pol Arch Intern Med 2019; 129: 808-17.

24. Korhonen MJ, Robinson JG, Annis IE, et al. Adherence tradeoff to multiple preventive therapies and all-cause mortality after acute myocardial infarction. J Am Coll Cardiol 2017; 70: 1543-54.

25. Urbinati S, Olivari Z, Gonzini L, et al. BLITZ-4 Investigators. Secondary prevention after acute myocardial infarction: drug adherence, treatment goals, and predictors of health lifestyle habits. The BLITZ-4 Registry. Eur J Prev Cardiol 2015; 22: 1548-56. 